\title{
Research and Design of Intelligent Network Switch Based on Human Infrared and Light Intensity Sensor
}

\author{
Yiqin Bao \\ School of Information Engineering, Nanjing Xiaozhuang University, Nanjing, China, 211171
}

\begin{abstract}
An intelligent network switch based on human infrared and light intensity sensors is designed by using human infrared sensor and light intensity sensor to realize the intelligent control of the home lamps. Switches will control the lights when there's only a man in the room and the light is dark, so as to achieve the goal of intelligent energy saving, and realize real-time data transmission under the condition of wireless networking. The switch takes the CC2530 RF chip as the core. The hardware and software of the node and sensor interface are designed in the ZigBee wireless sensor network. The whole system has advantages of good performance and high precision, and can meet the intelligent requirements of home lamps.
\end{abstract}

Keywords-CC2530; human infrared sensor; light sensor Introduction

\section{INTRODUCTION}

In this paper, we first discuss the current intelligent switching facilities, and provide a method of switching use today. In the last part, we compared with other traditional intelligent switch, put forward a new intelligent switch, using the human body infrared sensor light intensity sensor for intelligent control of lamps and lanterns[1] is in the home, and in the case of a network to realize real-time transmission of data.

Through the above steps, ensure that indoor lighting can be controlled accurately and efficiently. It has great help in the intelligent household control and management in the Internet of things.

\section{SYSTEM INFRASTRUCTURE AND PLATFORM}

\section{A. Intelligent Network Switch and Components}

Intelligent network switch designing. Intelligent network switch based on CC2530 chip. It supports 8KB RAM and builtin flash 256k memory, two series ports and twenty-one generic I/O pins. The CC2530 microcontrollers are based on a 8-bit and a real (Soc) solution for the 2.4-GHz 802.15.4, ZigBee and RF4CE applications. It can build strong network nodes with very low total material costs, that combine the microcontroller with 32/64/128/256 kb of embedded high-speed flash memory. A 16/24-bit MAC timer and a unique accelerator architecture enable 32-bit code to be executed at maximum clock frequency.

\section{B. Intelligent Network Switch Infrastructure}

The intelligent network switch mainly includes CC2530 chip, human infrared sensor, light sensor and relay, as shown in Figure 1. The intelligent network switch realizes the data communication through the radio frequency function of CC2530. Because of the 65000 nodes in the ZigBee network, the transmission of network data can be realized, so that the indoor lighting can be unified and controlled.

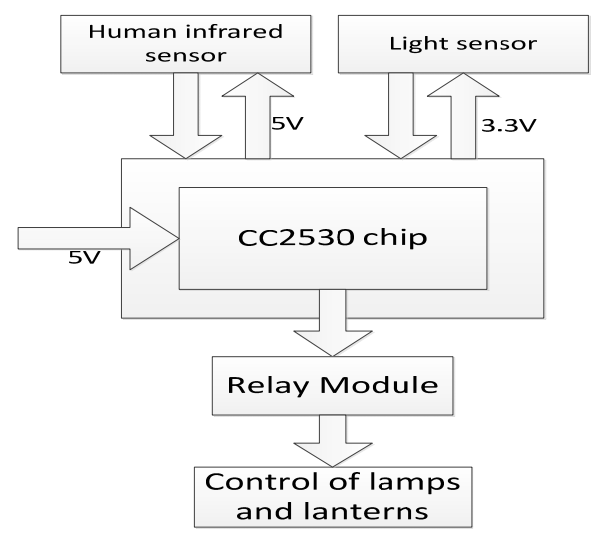

FIGURE I. INTELLIGENT NETWORK SWITCH

\section{SWITCH HARDWARE FRAMEWORK AND DESIGN}

\section{A. The Overview of Hardware}

The intelligent network switch based on human infrared and light sensor is mainly composed of CC2530 chip, human infrared sensor, light sensor and relay. Based on the original simple switch, the infrared sensor and light sensor are installed.

\section{B. Introduction of Human Infrared Sensor}

The infrared sensor of human body is the sensor with infrared as the medium, and also known as pyro electric sensor. The polarization state of piezoelectric ceramic dielectric after polarization is called spontaneous polarization. Spontaneous polarization decreases with temperature, and at Curie point, the temperature drops to zero. So when the material receives infrared radiation and the temperature increases, the surface load will decrease, which is equivalent to releasing some of the charge, which is the pyro electricity. The released charge can be converted to voltage output by the amplifier, which is how the pyro electric sensor works[3][4]. In the design, HC-SR501 human infrared sensor is used.

The infrared sensor of the human body can be detected within the range of measurable when the switch is being tested. When someone enters its induction range, the output is high level, and the person leaves the sensor range and automatically delays the high level and output low level CC2530 through its high and low level, the current detection range of whether anyone to make a decision. The state principle diagram of the infrared sensor is shown in Figure 2. 


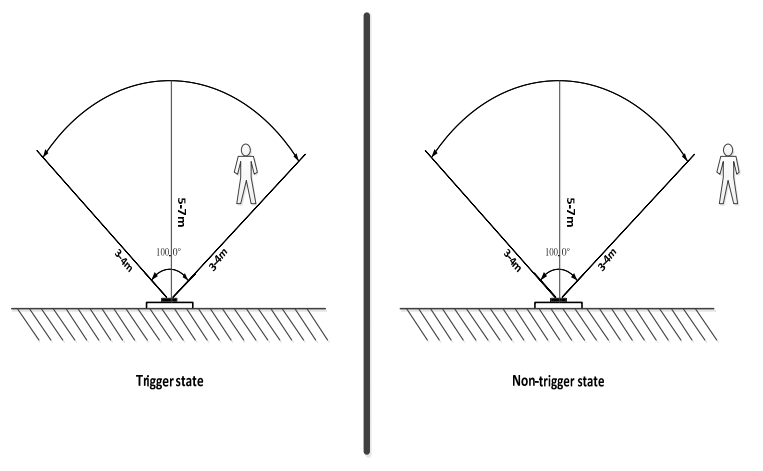

FIGURE II. SCHEMATIC DIAGRAM OF SENSOR STATE

The physical object is shown in Figure 3. The sensor has three pins that can be used to meet CC2530. The VCC is connected to the positive pole, the GND is connected to the negative pole, and the OUT connection is connected with the input port of CC2530. For this sensor, the sensitivity and delay time can be adjusted according to the actual situation through the sensor's knobs.

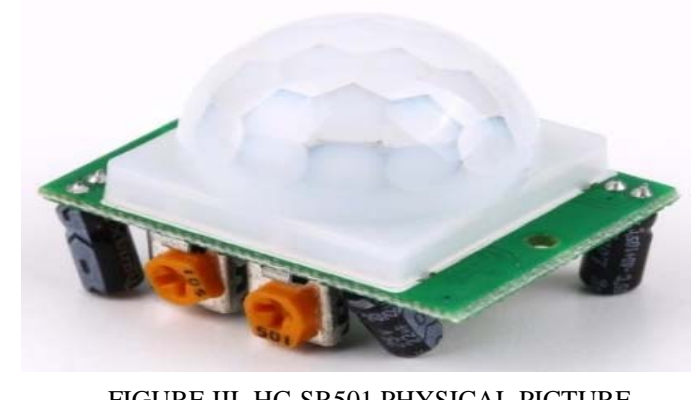

FIGURE III. HC-SR501 PHYSICAL PICTURE

\section{Introduction of Light Sensor}

Light sensor is a sensor that converts light intensity to voltage. Light intensity refers to the intensity of light. A measure of the energy of the visible light in a unit area, referred to as IL luminance (Lux or Lx). An object that is irradiated by light, the luminous flux obtained in the area of 1 square meter is 1 lumen, and his illumination is 1 lux. Lumens are units of luminous flux. Luminous intensity is a point light source with 1 candle, and the luminous flux emitted in the unit vertical Angle (1 stearin) is "1 lumen"[3][4]. The BH1750 light sensor is used in this design, and the internal circuit diagram is shown in the Figure 4.

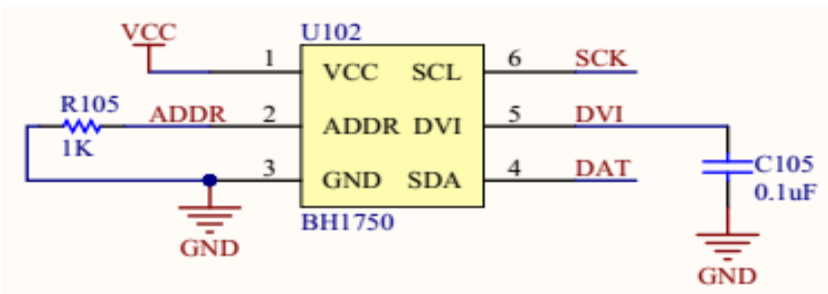

FIGURE IV. INTERNAL CIRCUIT DIAGRAM OF BH1750 LIGHT SENSOR

If the infrared sensor detects someone when the switch is in operation, the light sensor can collect the current light data of the room in real time, and convert the voltage value to CC2530. CC2530 determines the current light value by the voltage value transmitted by the light sensor. The working principle diagram is shown in Figure 5 .

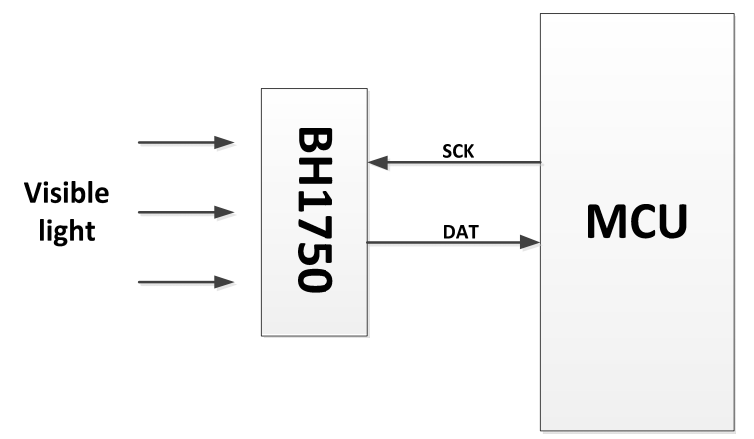

FIGURE V. ILLUMINATION SENSOR SCHEMATIC DIAGRAM

The actual figure is shown in figure 6. BH1750 is a digital optical intensity sensor integrated circuit for two-wire serial bus interface (IIC), so four different pins are used to connect to CC2530. VCC is connected to the positive pole, GND is connected to the negative pole, and SCK and DAT are connected to CC2530 input port respectively, as the clock signal end and data end of the I2C bus programming.

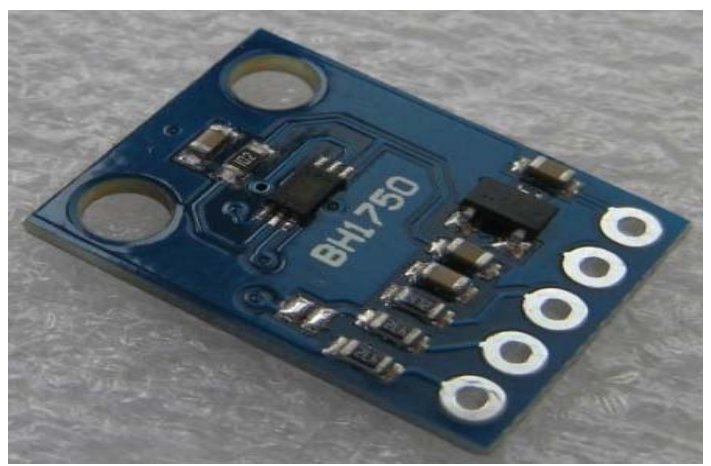

FIGURE VI. BH1750 PHYSICAL PICTURE

\section{Introduction of CC2530}

CC2530 is Texas Instruments (referred to as "TI) production, based on the $2.4 \mathrm{GHz}$, IEEE 802.15.4, ZigBee and RF4CE applications as a true on a chip (SoC) solution system. It can be established a powerful network nodes in a very low total cost of materials and carried out on the chip programming, so that perform the logic operation, sequence control, timing, counting and arithmetic operations etc. Environmental monitoring and equipment control signal output can be measured through digital or analog input/output. In the concrete implementation, CC2530F256 is selected for the implementation of greater function. The minimum system circuit diagram is shown in Figure 7. 


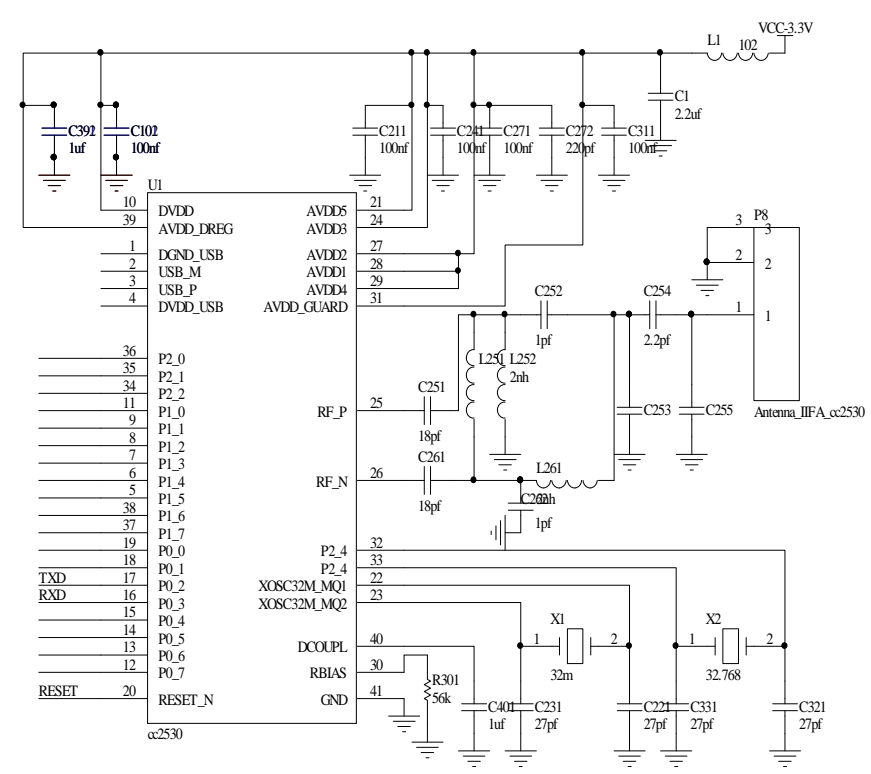

FIGURE VII. CIRCUIT DIAGRAM OF SYSTEM OF CC2530

The human infrared sensor, light sensor and relay are connected with CC2530 respectively. The access port is set in the program for input or output. Preliminary hypothesis: the design switch is installed in the room. When someone enters the room, the infrared sensor of the human body will transmit the high level to CC2530.After receiving the high level, the CC2530 collects the current house price data in real time through the illumination sensor. If the current light data is lower than the set threshold, CC2530 outputs a high level signal to the relay, so that turn on the light; instead, turn off the lights. CC2530 establish the star network, the coordinator receives all online terminal nodes, all switches in the network of intelligent network, so that all switches in the network are under control. The program flow chart of CC2530 is shown in Figure 8.

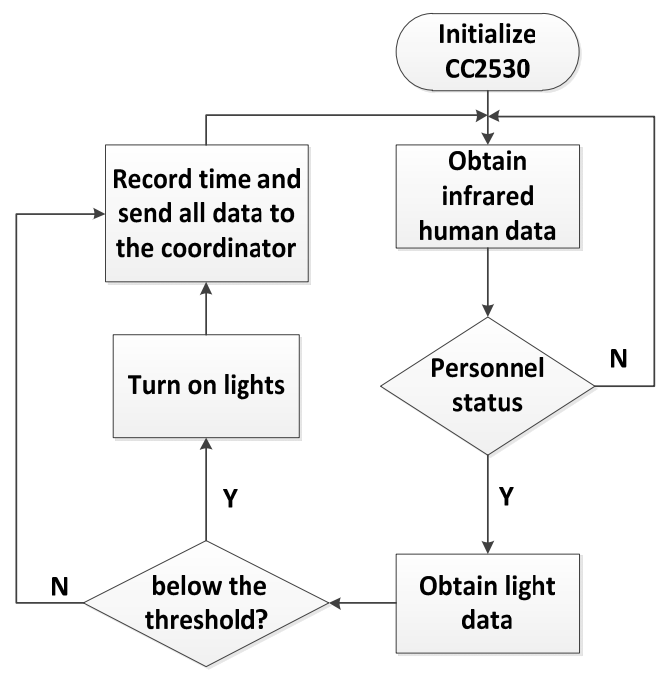

FIGURE VIII. PROGRAM FLOW CHART OF CC2530

\section{SWITCH SOFTWARE FRAMEWORK AND DESIGN}

\section{A. Switch Software Introduction}

Software platform include sensor data processing program and data sending program, sensor data processing program receive sensor data and perform analog to digital conversion. By Z-Stack, the sensor data, which has already processed can be transmitted in the wireless sensor network.

\section{B. File Transfer Protocol Format}

The data transfer process involves sensor data transmission, and it is important to specify the data frame format. The data protocol is shown in Table 1.

\section{TABLE I. DATA VERTICAL STRUCTURE}

\begin{tabular}{|c|c|}
\hline Field identification & Value meaning \\
\hline Beginning character & FFH \\
\hline Infrared sensor ID & $04 \mathrm{H}$ \\
\hline Sensor data & data \\
\hline Light intensity sensor ID & $06 \mathrm{H}$ \\
\hline Light data(L) & data(L) \\
\hline Light data(H) & data(H) \\
\hline Relay ID & 0FH \\
\hline Relay status & S \\
\hline Ending character & $00 \mathrm{H}$ \\
\hline
\end{tabular}

In above frame format, the sensor id, which determines the use of sensor categories, is the only identification for the distributed data sending and receiving.

\section{CONCLUSIONS}

The application of this intelligent switch makes the luminaire of the room be controlled intelligently. The user does not need to go to the switch to open or close the lamps when entering the room. The lights will automatically close regardless of the light is good during the day or no one in the room. In addition, because the switch is intelligent, it can also be more energy efficient and low carbonization. As a real piece of the system, the operation of CC2530 is stable, low power consumption, and not easy to damage; the program is stable and reliable, and the choice is good.

Compared with other single chips, CC2530 has the advantages of low power consumption, self-organizing network, strong stability and high reliability etc. The intelligent network switch based on human infrared and light intensity sensor has high application and promotion value.

\section{REFERENCES}

[1] Qian Zhihong,Wang Yijun. An overview of wireless sensor networks for the Internet of things [J] Journal of electronics information technology,2013,01:215-227.

[2] Zhu Hongbo,Yang Longxiang,Zhu Qi. Progress and application of Internet of things technology [J]. Journal of nanjing university of posts and telecommunications (natural science edition),2011,01:1-9

[3] Qian Zhihong,Wang Yijun. Internet of things technologies and application research [J].Chinese Journal of Electronics,2012,05:10231029.

[4] Bao Yiqin. Research and discussion on smart home system based on Internet of things technology,Internet of Things. 2013(7).

[5] 《The Principle of Hall Sensor's velocity measurement》》,Baidu Library, 2012. 
[6] Chen Xi, Xu Peng, Xu Xiaolei. Development of embedded industrial control system based on Bluetooth communication technology [J]. Instrument technology and sensor, 2015 (1): 74-76.

[7] Zhang Liang, Lingyun Feng, Wang Wen, Bi Tao. Design and implementation of experiment attendance system based on RFID [J]. Modern electronic technology, 2015 (1)

[8] Wang Weihong, Chen Xiaoliang. Design and implementation of mobile phone system based on wireless router[J]. Journal of Zhejiang University of Technology, 2016 (6).

[9] Jing Zhou, Quan Wei, Tang Jie, Yan Han. Sign-up system based on face recognition[J]. Software engineering, 2017 (5). 